\title{
Association of Patients' Perception of Quality of Healthcare Received and Colorectal Cancer Screening Uptake: An Analysis of 2 National Surveys in the USA
}

\author{
Karan Chawla ${ }^{a}$ Angesom Kibreab $^{b}$ Victor Scott $^{b}$ Edward L. Lee ${ }^{c}$ \\ Farshad Adulib Hassan Brim $^{c}$ Hassan Ashktorab ${ }^{b}$ Charles D. Howell ${ }^{b}$ \\ Adeyinka O. Laiyemo ${ }^{b}$ \\ aDepartment of Medicine, George Washington School of Medicine and Health Sciences, Washington, DC, USA; \\ bepartment of Medicine, Howard University College of Medicine, Washington, DC, USA; 'Department of \\ Pathology, Howard University College of Medicine, Washington, DC, USA
}

\section{Highlights of the Study}

- Patients must be given the opportunity to evaluate the quality of their healthcare.

- The perception of patients regarding the quality of healthcare services is associated with the likelihood of them following physician recommendations.

- An effort must be made to understand the characteristics of healthcare delivery that improve overall patient satisfaction.

\section{Keywords}

Colon cancer screening · Healthcare services ratings .

Colonoscopy · Fecal occult blood tests

\begin{abstract}
Objective: It is not known whether patients' ratings of the quality of healthcare services they receive truly correlate with the quality of care from their providers. Understanding this association can potentiate improvement in healthcare delivery. We evaluated the association between patients' ratings of the quality of healthcare services received and uptake of colorectal cancer (CRC) screening. Subjects and Methods: We used 2 iterations of the Health Information National Trends Survey (HINTS) of adults in the USA. HINTS 2007
\end{abstract}

$(4,007$ respondents; weighted population $=75,397,128)$ evaluated whether respondents were up to date with CRC screening while HINTS 4 cycle 3 (1,562 respondents; weighted population $=76,628,000$ ) evaluated whether participants had ever received CRC screening in the past. All included respondents from both surveys were at least 50 years of age, had no history of CRC, and had rated the quality of healthcare services that they had received at their healthcare provider's office in the previous 12 months. Results: HINTS 2007 data showed that respondents who rated their healthcare as

Abstracts from this study were presented at the Digestive Diseases Week in 2016 in San Diego (Gastrointest Endosc. 2016;83(5):AB449) and at the Digestive Diseases Week in Washington, DC, in June 2018 (Gastrointest Endosc. 2018;87(6):AB384).
C 2020 The Author(s)

Published by S. Karger AG, Basel

This is an Open Access article licensed under the Creative Commons Attribution-NonCommercial-4.0 International License (CC BY-NC) (http://www.karger.com/Services/OpenAccessLicense), applicable to the online version of the article only. Usage and distribution for commercial purposes requires written permission.
Correspondence to:

Adeyinka O. Laiyemo, adeyinka.laiyemo@howard.edu 
good or fair/poor were significantly less likely to be up to date with CRC screening compared to those who rated their healthcare as excellent. We found comparable results from analysis of HINTS 4 cycle 3 data with poorer uptake of CRC screening as the healthcare quality ratings of respondents reduced. Conclusion: Our study suggests that patients who reported receiving lower quality of healthcare services were less likely to have undergone and be compliant with CRC screening recommendations. It is important to pay close attention to patient feedback surveys in order to improve healthcare delivery.

(c) 2020 The Author(s)

Published by S. Karger AG, Basel

\section{Introduction}

Colorectal cancer (CRC) is one of the leading causes of cancer-related deaths in the USA, with approximately 50,000 deaths occurring annually [1]. CRC screening guidelines have recommended the use of fecal occult blood testing (FOBT), fecal immunochemical testing, sigmoidoscopy, and colonoscopy to screen asymptomatic average-risk adults between the ages of 50 and 75 to reduce the burden of the disease [2-5]. Although screening rates have been steadily increasing in the USA in the last 2 decades, there is a great need for substantial improvement in order to reduce the burden from this potentially preventable disease [6]. The National Colorectal Cancer Roundtable (NCCR) set a goal of achieving $80 \%$ adherence to CRC screening rates in the USA by 2018 [7]. However, currently one out of every 3 eligible adults is not up to date with CRC screening guideline recommendations [8].

The recommendation from a care provider to their patients is among the strongest predictors of undergoing CRC screening [9]. Studies have shown that communication and level of trust of the healthcare provider are associated with increased CRC screening uptake [10-14]. In recent years, there has been a focus on improving the quality of healthcare delivery using feedback from patient satisfaction surveys such as Press Ganey Survey. These surveys are typically filled out by patients after they have received healthcare services [15].

The implications of improved customer satisfaction are well known in business management, and efforts are constantly being made to gather this information as well as improve customers' experience since it improves loyalty. Customer loyalty is central to business success of healthcare providers and impacts health outcomes [16]. This understanding is being imported rapidly into healthcare delivery [17-19]. For example, patients tend to perceive higher quality of healthcare in private than in public healthcare facilities [19]. Patient feedback surveys are increasingly being used as a component of performance evaluation of care providers [20-22], and many organizations have financial incentives for performance.

We hypothesize that patients who opined that they have been receiving quality healthcare services are more likely to have been screened for CRC. In this study, we used 2 publicly available national data to evaluate the uptake of CRC screening as a function of healthcare quality perception and ratings by patients.

\section{Subjects and Methods}

\section{Study Population}

After obtaining approval from the Institutional Review Board of Howard University in Washington, DC (IRB-14-MED-28), we downloaded the datasets. We used data from the Health Information National Trends Survey (HINTS) 2007 and HINTS 4 cycle 3 iterations because both inquired about the exposure of interest and were conducted about 6 years apart with different respondents.

\section{HINTS 2007}

The details of HINTS 2007 have been published by Cantor et al. [23]. The data in the HINTS 2007 were collected by conducting random digital dial phone interviews and by mailing surveys to households listed by the US Postal Service. Phone interviews were conducted in English or Spanish based on the preferred language of communication of the respondents. Households that were sent surveys via mail were sent a survey initially, followed by 2 more mailing cycles if they did not respond. Households that still did not respond were then provided with a final reminder via phone. A phone survey was noted to be complete if the interviewee participated in the survey to completion, partially complete if at least the first part of the survey (Health Communications) was completed, and invalid if the first part of the survey was not completed. A mailed survey was categorized as complete if at least $80 \%$ of the questions were answered, partially complete if $50-79 \%$ of the questions were answered, and invalid if $<50 \%$ of the questions were answered. The overall response rate was $24.2 \%$ for the phone survey and $31 \%$ for the mail survey. There were 4,092 responses to the phone survey and 3,582 responses to the mail survey, totaling to 7,674 responses in all. Survey respondents were asked questions regarding their adherence to CRC screening with FOBT, sigmoidoscopy, and colonoscopy, as well as when these tests were performed to ascertain if they were up to date with CRC screening guidelines. They were also asked to rate the quality of healthcare services that they had received in the previous 12 months with the question "Overall, how would you rate the quality of health care you received in the past 12 months?" The respondents chose an answer from the following choices: "excellent," "very good," "good," "fair," and "poor." 
Respondents were excluded from this study if they were $<50$ years of age, if they had a history of CRC, or if they did not answer questions regarding healthcare quality and CRC screening. Our final analytical data included 4,007 survey respondents (weighted population size of $75,397,128)$.

\section{HINTS 4 Cycle 3}

HINTS 4 cycle 3 was conducted via mail over a 4 -month span in 2013. The details of the survey have been published by Finney Rutten et al. [24]. Four mailing cycles were done for this survey, with all households included in the first mailing cycle and nonrespondents included in the subsequent 3 mailing cycles. Households that were flagged as having Spanish-speaking occupants received English and Spanish versions of the survey. Identical to the mailed-in HINTS 2007, the HINTS 4 cycle 3 was categorized as complete if at least $80 \%$ of the questions were answered, partially complete if $50-79 \%$ of the questions were answered, and invalid if $<50 \%$ of the questions were answered. The survey had a response rate of $35.2 \%$ and included 3,185 respondents. In this study, survey respondents were also asked to rate the quality of healthcare received in the previous 12 months like was done for the HINTS 2007. They were also asked whether they ever had a colonoscopy, sigmoidoscopy, or stool blood test to check for colon cancer regardless of when the examinations took place. We included respondents who were at least 50 years old without personal history of CRC who responded to the questions regarding the quality of healthcare services they received and history of CRC screening. Therefore, our analytical cohort consisted of 1,562 respondents (weighted population size of $76,628,000)$.

\section{Statistical Analysis}

We performed separate analyses of the datasets because the outcomes being investigated were different. The outcome of interest was being up to date with CRC screening in HINTS 2007 which was defined as the uptake of FOBT within 1 year, sigmoidoscopy within 5 years, or colonoscopy within 10 years. For HINTS 4 cycle 3 , the outcome of interest was whether respondents had ever been screened for CRC with FOBT, sigmoidoscopy, or colonoscopy regardless of the time that the screening took place.

For the purpose of our data analysis, the responses of "fair" and "poor" were grouped into 1 category given the fact that "fair" designation is generally considered to be of suboptimal quality. Furthermore, there were low number of responses in these 2 categories. We used survey weights and compared the demographic and lifestyle characteristics of survey respondents in the 2 datasets by their ratings of the quality of healthcare they received using $\chi^{2}$ tests for categorical variables and noted the $p$ value of the comparisons. We calculated the percentage of respondents who were up to date with CRC screening (HINTS 2007) and those who reported ever being screened for CRC (HINTS 4 cycle 3 ) by their ratings of the quality of healthcare services they received. Survey weights were used in all data analyses, and Taylor series linearization was used for variance estimations to account for the complex survey design. Binary logistic regression analyses were used to estimate odds ratios (OR) and 95\% confidence intervals (CI). The final models included age, sex, smoking status, highest education level, marital status, health insurance coverage status, body mass index, and race. Age was included in the model as a continuous variable. We used Stata statistical software version 14.2 (College Station, TX,
USA) for all analyses. All reported percentages were weighted, and a $p$ value $<0.05$ was considered as statistically significant and bold in the tables.

\section{Results}

\section{Characteristics of HINTS 2007 Respondents}

A total of 4,007 respondents (weighted population size of $75,397,128$ ) were included for the HINTS 2007 component of the study. The mean age was 64.1 years $(95 \% \mathrm{CI}$ : 63.8-64.3 years), and $54.6 \%$ of the respondents were females. The characteristics of survey respondents by how they rated the quality of their healthcare in the previous 12 months are shown in Table 1. Of all respondents, $37.1 \%$ rated their quality of healthcare as excellent, $40.9 \%$ rated it as very good, $16.6 \%$ rated it as good, and $5.4 \%$ rated it as fair or poor. Respondents in the youngest age group (50-64 years) were more likely to report receiving fair/poor healthcare $(p=0.0481)$. Caucasian respondents were most likely to report excellent healthcare while Hispanic respondents reported the highest fair/poor healthcare ratings $(p<0.001)$. Respondents with higher levels of education were also more likely to report receiving excellent healthcare $(p=0.002)$. Current smokers had higher rates of fair/poor healthcare compared to respondents who had never smoked or were former smokers $(p<$ $0.001)$. Those without insurance coverage were more likely to report fair/poor healthcare than those with healthcare insurance $(p<0.001)$. Finally, patients who were not married were more likely to report having fair/ poor healthcare than those who were married $(p=0.002)$.

\section{Characteristics of HINTS 4 Cycle 3 Respondents}

There were 1,562 respondents (weighted population size $=76,628,000)$ in the HINTS 4 cycle 3 component of the study. The mean age was 64.2 (95\% CI: 63.8-64.5) years, and $55.4 \%$ of the respondents were females. Table 2 displays the characteristics of respondents by their rating of the quality of healthcare services that they received in the previous 12 months. Of all respondents, 39.0\% rated their quality of healthcare as excellent, $37.8 \%$ rated it as very good, $17.6 \%$ rated it as good, and 5.6\% rated it as either fair or poor. Similar to trends seen in the HINTS 2007 data, patients in the youngest age category were most likely to report experiencing fair/poor healthcare services $(p=0.048)$. Hispanic patients, current smokers, and those without healthcare insurance were more likely to report fair/poor healthcare services. 
Table 1. Characteristics of respondents to HINTS $2007(N=4,007$; weighted population size $=75,397,128)$

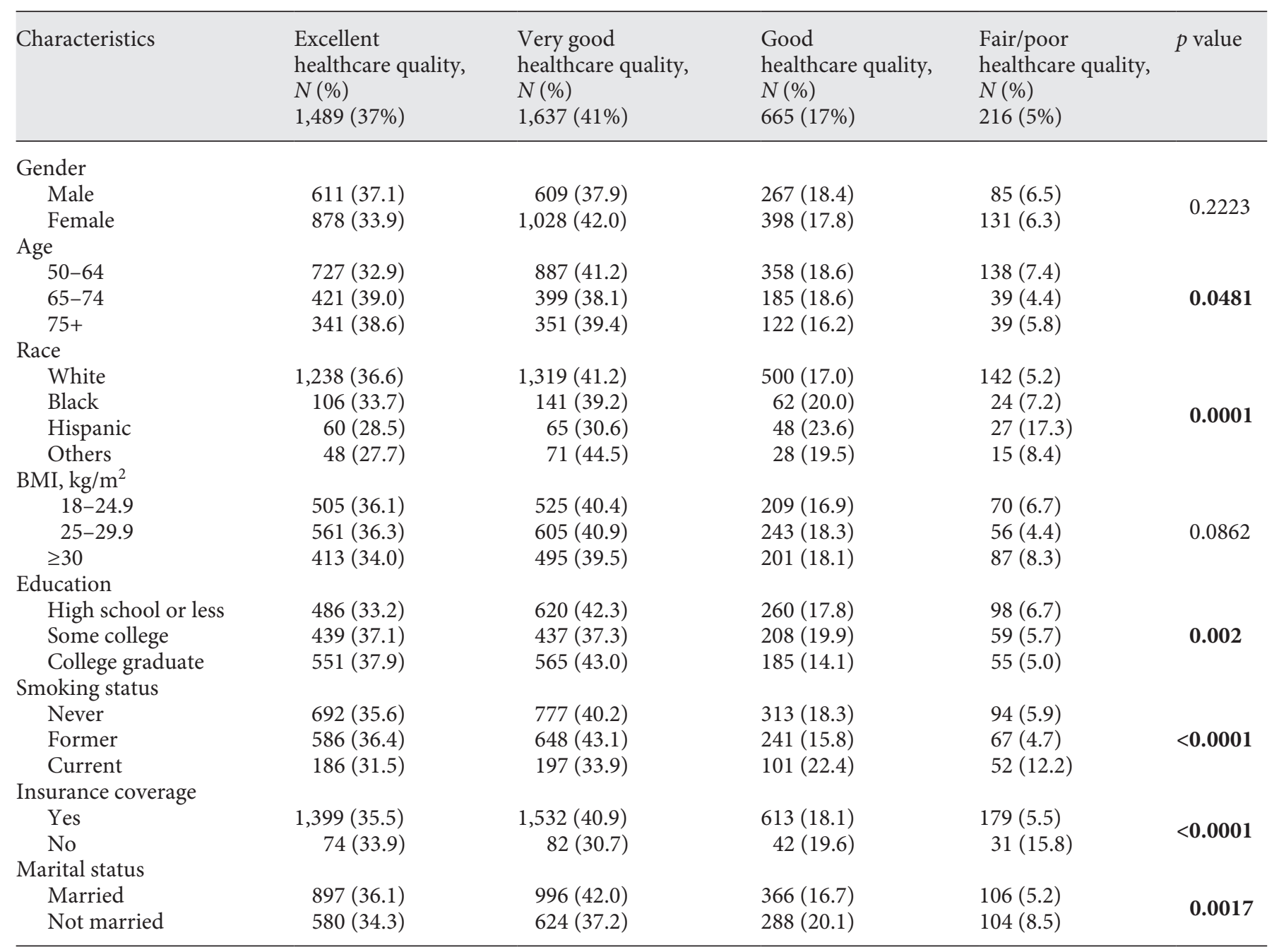

Missing data: race $=113, \mathrm{BMI}=37$, education $=44$, smoking status $=53$, insurance coverage $=55$, and marital status $=46$. Bold $p$ values are significant.

Up to Date with CRC Screening by Healthcare Rating (HINTS 2007)

In the HINTS 2007 component of the study, $69.6 \%$ of the respondents with the excellent healthcare rating, $67.4 \%$ in the very good category, $59.8 \%$ in the good category, and $54.8 \%$ in the fair/poor category were up to date with CRC screening (Table 3). Overall, analysis showed that the likelihood of being up to date with CRC screening decreased as the quality of healthcare services ratings decreased. For those who rated their healthcare services received as very good, there was no statistically significant difference from those who rated the quality of their healthcare services as excellent in the univariate analysis and after adjusting for covariates in the multivariable models. However, there was statistically significant decreased compliance with CRC screening guidelines for respondents who rated the quality of their healthcare services as good or fair/poor.

\section{Ever Screened for CRC by Healthcare Rating (HINTS 4 Cycle 3)}

In the HINTS 4 cycle 3 component of our study, $83.0 \%$ of the respondents with excellent healthcare rating, $74.4 \%$ in the very good category, $68.1 \%$ in the good category, and $59.0 \%$ in the fair/poor category had undergone CRC screening previously (Table 4 ). We found a pattern that was similar to being up to date with CRC screening in HINTS 2007 data. The lower the rating of the quality of 
Table 2. Characteristics of respondents to HINTS $2007(N=4,007$; weighted population size $=75,397,128)$

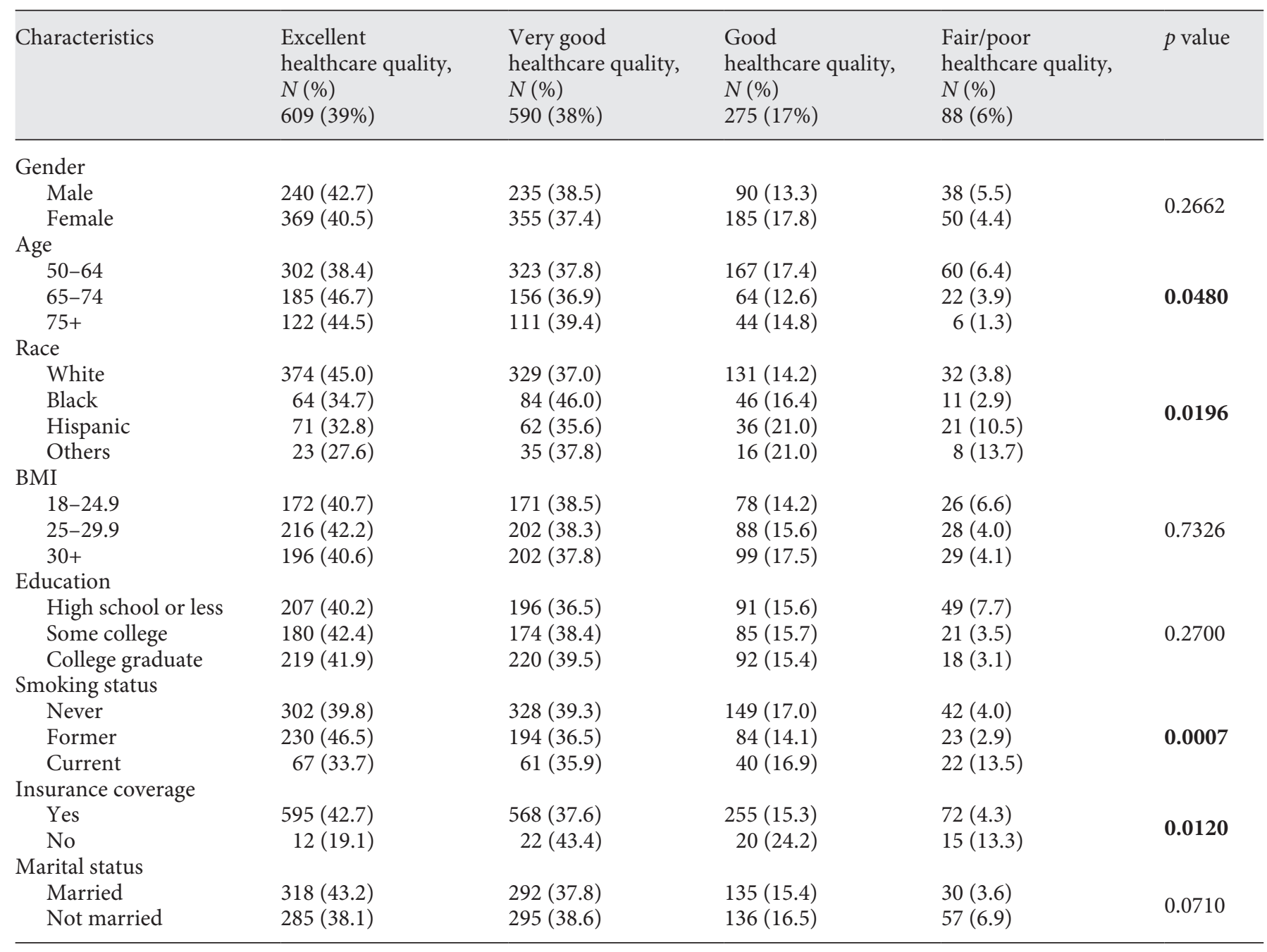

Missing data: race $=219, \mathrm{BMI}, 55$, education $=10$, smoking status $=20$, insurance coverage $=3$, and marital status $=14$. Population size $=76,628,000$. Bold $p$ values are significant.

Table 3. Association of healthcare quality rating with up to date with CRC screening (HINTS 2007)

\begin{tabular}{lccc}
\hline Ratings of healthcare services received $(N)$ & \multicolumn{2}{l}{ Up to date with CRC screening } & \\
\cline { 2 - 4 } & $n($ wt $\%)$ & univariate OR (95\% CI) & multivariate OR (95\% CI) ${ }^{\text {a }}$ \\
\hline Excellent $(1,489)$ & $1,075(69.6)$ & Reference & Reference \\
Very good $(1,637)$ & $1,128(67.4)$ & $0.90(0.75-1.09)$ & $0.95(0.78-1.16)$ \\
Good (665) & $415(60.0)$ & $0.65(0.52-0.81)$ & $0.70(0.54-0.90)$ \\
Fair/poor $(216)$ & $113(54.8)$ & $0.53(0.38-0.74)$ & $0.66(0.46-0.96)$ \\
\hline
\end{tabular}

CRC, colorectal cancer; OR, odds ratio; CI, confidence intervals. ${ }^{\text {a }}$ Adjusted for age, sex, smoking status, highest education level, marital status, health insurance coverage status, BMI, and race. 
Table 4. Association of healthcare quality rating with ever screened for CRC (HINTS 4 cycle 3)

\begin{tabular}{llll}
\hline $\begin{array}{l}\text { Ratings of healthcare } \\
\text { services received }(N)\end{array}$ & \multicolumn{2}{l}{ Ever screened for CRC } & \\
\cline { 2 - 4 } & $n(\mathrm{wt} \%)$ & univariate OR (95\% CI) & multivariate OR $(95 \% \text { CI })^{\mathrm{a}}$ \\
\hline Excellent $(609)$ & $510(83.0)$ & Reference & Reference \\
Very good $(590)$ & $456(74.4)$ & $0.59(0.36-0.98)$ & $0.61(0.35-1.07)$ \\
Good (275) & $193(68.1)$ & $0.44(0.28-0.69)$ & $0.52(0.31-0.89)$ \\
Fair/poor $(88)$ & $47(59.0)$ & $0.29(0.15-0.58)$ & $0.43(0.16-1.17)$ \\
\hline
\end{tabular}

CRC, colorectal cancer; OR, odds ratio; CI, confidence intervals. a Adjusted for age, sex, smoking status, highest education level, marital status, health insurance coverage status, BMI, and race.

healthcare services received, the lower the likelihood of ever been screened for CRC previously. Analysis showed that those who rated their healthcare services as very good had lower but nonstatistically significant differences in the likelihood of ever been screened for CRC. However, those who rated their healthcare as good had significant $48 \%$ reduced odds of ever been screened for CRC when compared to those with excellent ratings for their healthcare services. However, we noted statistically significant reduced odds of screening among respondents who rated the quality of their healthcare services as fair/poor in the univariate model, but the $57 \%$ reduced odds in the multivariate model was not statistically significant due to small numbers in the cells after adjustment for covariates.

\section{Discussion}

Although surveys on patients' satisfaction are often employed to assess the quality of healthcare delivery, it is not known whether patients' ratings of the quality of the healthcare services actually predict important quality health outcomes. In this study, we used 2 iterations of the Health Interview National Trends Survey data (HINTS 2007 and HINTS 4 cycle 3) to evaluate whether the ratings of the quality of healthcare services received by patients are associated with uptake of CRC screening. In these 2 nationally representative data, we found a direct correlation between high-quality ratings of healthcare services received in the previous 12 months and compliance with colon cancer screening. We noted that respondents who rated their quality of healthcare higher were more likely to have had CRC screening at some point in the past and were also more likely to be up to date with CRC screening recommendations. These findings suggest that patients who are more satisfied with the quality of healthcare that they are receiving have a greater propensity to adhere to
CRC screening guidelines. This underscores the importance of patient satisfaction surveys and the need to obtain feedback from patients regarding their healthcare services.

The implication of our study is that patients' ratings of the quality of healthcare services may be an accurate reflection of the quality of healthcare services they actually receive especially in preventive healthcare services and CRC screening in particular. Although the reason why the respondents were motivated to undergo CRC screening was not explored in both surveys, we speculated that respondents who felt that they were receiving high-quality healthcare services were more likely to follow the recommendations of their care providers.

We are not aware of any study that has investigated the association between patients' perception and overall ratings of the quality of their healthcare services and their likelihood of undergoing CRC screening for a direct comparison with our study. However, Katz et al. [14] investigated the association between patient-provider communication and the patient's likelihood of undergoing CRC screening. The study consisted of a telephone survey that was completed by 397 church members at 12 AfricanAmerican churches in North Carolina. The authors reported that study participants who were deemed to have experienced "good" communication with their physicians had a higher likelihood of being up to date with CRC screening compared to the "poor" communication group (35.9 vs. $16.7 \% ; p=0.013$ ) [14].

In another study, Gupta et al. [11] examined the relationship between patients' trust in healthcare providers and CRC screening uptake among an ethnically diverse set of patients with low income participating in a randomized clinical trial in San Francisco. The Wake Forest Trust Scale was used to measure the level of trust that the patients had in their primary care physicians and in doctors in general. The participants were considered to have 
adhered to their primary care providers' recommendations if they received the recommended CRC screening tests within 12 months of enrollment into the trial. The investigators reported that trust in their primary care providers, but not trust in doctors in general, was associated with an increased likelihood of completing their CRC screening as recommended (OR: 1.11; 95\% CI: 1.031.17). In another study, Born et al. [12] also examined the relationship between patient trust in their physicians and CRC screening. Although the study focused only on the FOBT screening modality, the authors also reported that participants who completed the FOBT as recommended had a higher level of trust in their physician compared to those who did not [12]. These suggest that the special professional relationship between patients and their specific care providers influences their compliance with healthcare recommendations and underscores the need to improve trust of patients in their healthcare providers. Our study also suggests that the level of patients' satisfaction with their healthcare providers is associated with the likelihood of adhering to physician recommendations. We postulate that the more trust the patients have in their healthcare providers, the better their perception of the quality of services will be and may positively influence the compliance of the patients with the healthcare recommendations they receive including CRC screening. It is important to obtain feedback from patients and make changes to improve patients' satisfaction towards achieving quality improvement in healthcare institutions [15].

A proposed model for obtaining important patients' feedback suggests obtaining information regarding tangibility, reliability, responsiveness, assurance, and empathy during their encounters with healthcare providers. By understanding discrepancies between the expected level of care in these 5 fields and the level of care observed by patients, an effort can be made to gear improvements specifically towards areas of weakness in provision of care [25]. Another model for assessment of care quality is the use of a psychometric scale called Service Quality (SERVQUAL). This is an instrument consisting of 44 questions that allows patients to convey their expected and perceived quality of care attained during a healthcare encounter. The SERVQUAL instrument has been used in various nations across a number of medical subspecialties and has served as a vital tool in detecting shortfalls in care offered [25]. Furthermore, Reichheld's ultimate question is also considered to be an effective tool for collecting patient satisfaction data in a simple and inexpensive manner. In addition, it also takes staff satisfaction into consideration, with the understanding that staff satisfaction ultimately affects patients' experience. The tool consists of 1 primary

Healthcare Quality Perception and

Colorectal Cancer Screening question: "On a scale of $0-10$, how likely are you to recommend a product or service to a colleague, family member, or friend?" as well as a follow-up question that states "What one or two things could we do to increase your rating to a 9 or a 10 ?" A key difference between the ultimate question tool and other traditional tools is that the follow-up question in the ultimate question tool is open ended, allowing patients to convey their thoughts without being forced to use preset answer choices. This enables a more detailed and actionable patient survey answers [17].

The tools presented above, among others that serve a similar purpose, must be employed in the clinical setting to understand specifically how the quality of care that we deliver to our patients can be improved. Patients' input will also allow for personalization of care. This will assist in improving the quality of care nationwide and on a global scale [26].

The strengths of our study are that we used 2 separate iterations of the HINTS conducted 6 years apart in 2007 and 2013, and the results were consistent that quality ratings by patients are associated with their compliance with CRC screening. The surveys were also conducted in English and Spanish, thereby reaching a diverse population in the country. Furthermore, the 2007 survey was conducted by mail and by phone in order to reach those without a registered mailing address. However, our study has certain limitations. Firstly, our data were based on selfreports, and the deidentified nature of the data meant that the respondents could not be reached to obtain their consent for actual review of their medical records. Hence, it is quite possible that some respondents who had an endoscopy for diagnostic reasons may have reported having these tests for the purpose of CRC screening. Nonetheless, patients who underwent colonoscopy for diagnostic purposes would still have received the screening advantages of the procedure. Secondly, we were unable to delineate the specific reasons that respondents who reported receiving fair or poor healthcare services were unhappy with the services they received. Thirdly, we did not have sufficient details about the type of healthcare service utilized by the respondents, details of their insurance coverage, and the specific specialty of their care providers.

\section{Conclusion}

The analysis of HINTS 2007 and HINTS 4 cycle 3 revealed that patients who opine that they received excellent healthcare services were more likely to have ever been screened for CRC and were more likely to be up to date 
with CRC screening guidelines. The implication of this finding is that care providers should actively engage in conversations with their patients to understand exactly how to improve the quality of healthcare services that the patients receive. Further research is needed to better understand what specific characteristics of healthcare services delivery patients seek in order to consider their healthcare services to be of high quality.

\section{Statement of Ethics}

The Institutional Review Board of Howard University in Washington, DC (IRB-14-MED-28), approved the study as exempt. The study used publicly available deidentified data.

\section{Conflict of Interest Statement}

We have no conflicts of interest to report.

\section{Author Contributions}

Planning and/or conducting the study: K.C., H.B., H.A., and A.O.L.; collecting and/or interpreting the data, drafting/revision of the manuscript, and approval of the final draft of the submitted manuscript: K.C., A.K., V.S., E.L.L., F.A., H.B., H.A., C.D.H., and A.O.L.

\section{References}

1 Siegel RL, Miller KD, Fedewa SA, Ahnen DJ, Meester RGS, Barzi A, et al. Colorectal cancer statistics, 2017. CA Cancer J Clin. 2017 May; 6(67):177-93.

2 Rex DK, Boland CR, Dominitz JA, Giardiello FM, Johnson DA, Kaltenbach $\mathrm{T}$, et al. Colorectal cancer screening: recommendations for physicians and patients from the U.S. Multi-Society Task Force on Colorectal Cancer. Gastrointest Endosc. 2017 Jul;86(1):1833.

3 US Preventive Services Task Force; BibbinsDomingo K, Bibbins-Domingo K, Grossman DC, Curry SJ, Davidson KW Jr, et al. Screening for colorectal cancer: US Preventive Services Task Force recommendation statement. JAMA. 2016 Jun 21;315(23):2564-75.

4 U.S. Preventive Services Task Force. Screening for colorectal cancer: U.S. Preventive Services Task Force recommendation statement. Ann Intern Med. 2008 Nov 4;149(9):627-37.

5 Lieberman DA, Rex DK, Winawer SJ, Giardiello FM, Johnson DA, Levin TR. Guidelines for colonoscopy surveillance after screening and polypectomy: a consensus update by the US Multi-Society Task Force on Colorectal Cancer. Gastroenterology. 2012 Sep;143(3): 844-57.

6 Meissner HI, Breen N, Klabunde CN, Vernon SW. Patterns of colorectal cancer screening uptake among men and women in the United States. Cancer Epidemiol Biomarkers Prev. 2006 Feb;15(2):389-94.

7 Meester RG, Doubeni CA, Zauber AG, Goede SL, Levin TR, Corley DA, et al. Public health impact of achieving $80 \%$ colorectal cancer screening rates in the United States by 2018 . Cancer. 2015 Jul 1;121(13):2281-5.

8 Simon K. Colorectal cancer development and advances in screening. Clin Interv Aging. 2016 Jul 19;11:967-76.
9 Laiyemo AO, Adebogun AO, Doubeni CA, Ricks-Santi L, McDonald-Pinkett S, Young $\mathrm{PE}$, et al. Influence of provider discussion and specific recommendation on colorectal cancer screening uptake among U.S. adults. Prev Med. 2014;67:1-5.

10 Gao G, Burke N, Somkin CP, Pasick R. Considering culture in physician-patient communication during colorectal cancer screening. Qual Health Res. 2009 Jun;19:778-89.

11 Gupta S, Brenner AT, Ratanawongsa N, Inadomi JM. Patient trust in physician influences colorectal cancer screening in low-income patients. Am J Prev Med. 2014 Oct;47(4):417-23.

12 Born W, Engelman K, Greiner KA, Bhattacharya SB, Hall S, Hou Q, et al. Colorectal cancer screening, perceived discrimination, and low-income and trust in doctors: a survey of minority patients. BMC Public Health. 2009 Sep 25;9:363.

13 O'Malley AS, Sheppard VB, Schwartz M, Mandelblatt J. The role of trust in use of preventive services among low-income African-American women. Prev Med. 2004 Jun;38:777-85.

14 Katz ML, James AS, Pignone MP, Hudson MA, Jackson E, Oates V, et al. Colorectal cancer screening among African American church members: a qualitative and quantitative study of patient-provider communication. BMC Public Health. 2004 Dec 15;4:62.

15 Al-Abri R, Al-Balushi A. Patient satisfaction survey as a tool towards quality improvement. Oman Med J. 2014 Jan;29(1):3-7.

16 Zhou WJ, Wan QQ, Liu CY, Feng XL, Shang SM. Determinants of patient loyalty to healthcare providers: an integrative review. Int $J$ Qual Health Care. 2017;29(4):442-9.

17 York AS, McCarthy KA. Patient, staff and physician satisfaction: a new model, instrument and their implications. Int J Health Care Qual Assur. 2011 Feb;24(2):178-91.
18 Kessler DP, Mylod D. Does patient satisfaction affect patient loyalty? Int J Health Care Qual Assur. 2011;24(4):266-73.

19 Ahmed S, Tarique KM, Arif I. Service quality, patient satisfaction and loyalty in the Bangladesh healthcare sector. Int J Health Care Qual Assur. 2017;30(5):477-88.

20 Dryden EM, Hyde JK, Wormwood JBWu JCalloway RCutrona SL, et al. Assessing patients' perceptions of clinician communication: acceptability of brief point-of-care surveys in primary care. [published online ahead of print, 2020 Aug 3]. J Gen Intern Med. 2020; 35(10):2990-9.

21 Berger R, Bulmash B, Drori N, Ben-Assuli O, Herstein R. The patient-physician relationship: an account of the physician's perspective. Isr J Health Policy Res. 2020;9:33.

22 Rosenthal MB, Landon BE, Normand SL, Frank RG, Epstein AM. Pay for performance in commercial HMOs. N Engl J Med. 2006; 355(18): 1895-902.

23 Cantor D, Coa K, Crystal-Mansour S, Davis T, Dipko S, Sigman R. Health Information National Trends Survey (HINTS) 2007: final report. 2009.

24 Finney Rutten LJ, Davis T, Beckjord EB, Blake $\mathrm{K}$, Moser RP, Hesse BW. Picking up the pace: changes in method and frame for the health information national trends survey (20112014). J Health Commun. 2012;17(8):979-89.

25 Pena MM, da Silva EMS, Tronchin DMR, Melleiro MM. The use of the quality model of Parasuraman, Zeithaml and Berry in health services. Rev Esc Enferm USP. 2013;47(5): 1227-32

26 Delivering quality health services: a global imperative for universal health coverage. Geneva: World Health Organization, Organization for Economic Co-operation and Development, and The World Bank; 2018. 